\title{
Breve estudio del comportamiento ciudadano en calles de la Habana
}

\author{
Brief study of citizen behavior in Havana streets
}

Recibido: junio 2020

Aceptado: febrero 2021

\section{Resumen}

El artículo valora la importancia de las ciudades en la educación de las prácticas ciudadana. Expone la consideración de que en la actualidad la ciudad es vista como un actor de educación de las personas y que han aparecido nuevas ciencias que se ocupan de esta relación como la Pedagogía Urbana y la Neuroarquitectura. Además, hay cambio en las concepciones arquitectónicas para ir más a lo cualitativo y subjetivo del diseño de los lugares físicos de la ciudad. Expone la riqueza de los espacios públicos para los comportamientos ciudadanos y la necesidad de que su diseño tome en cuenta los usos que las personas le darán. Se ofrecen datos registrados en observaciones realizadas a 100 sujetos que practicaban el cruce de calles concurridas en La Habana. Ellos muestran que los sujetos cuidan su vestimenta e higiene. La mayoría cumple las reglas de esos espacios, lo que significa que han asimilado las practicas adecuadas. Otro por ciento muestra no cumplir esas reglas y por tanto requiere aumentar su formación ciudadana. El diseño físico de los espacios públicos tiene algunos objetos construidos que obstaculizan la movilidad y seguridad de las personas. Las prácticas ciudadanas ofrecen características típicas e interesantes de los sujetos.

\section{Palabras Claves:}

comportamiento ciudadano; espacio público; prácticas ciudadanas

\author{
Berta Margarita González Rivero ${ }^{1}$
}

The article shows the importance of cities in citizen practices education. It exposes that actually the conceptions of design change in order to include the qualities and subjectivities aspects of citizen's physicals spaces. It is necessary that these designs take people uses. It offers points of observations realized to 100 persons that are crossing the street in Havana city. They show that this people take care their appearance and hygienic body. Many of them keep the rules of the public spaces, but other ones do not keep them. All these citizen behaviors show typical and interest characteristics of people.

\section{Keywords:}

citizen practices; citizen behavior; public spaces

\footnotetext{
${ }^{1}$ Nacionalidad: cubana; adscripción: Universidad de La Habana; Doctora en Ciencias Psicológicas, Profesora Principal del Centro para el Perfeccionamiento de la Educación Superior (CEPES); correo: berta@cepes.uh.cu
} 


\section{INTRODUCCIÓN}

Las personas conviven en determinados grupos que se constituyen en agentes socializadores, tales como la familia, la escuela, el trabajo, etc. No obstante, ellas también viven en espacios públicos que, aunque están habitados no constituyen grupos de personas tal como se conocen. Esos son los espacios públicos en los que se interrelacionan no solo con muchas personas sino con un entorno diseñado. Son espacios de encuentros, debates, relaciones, intercambios, que tienen sus reglas no previstas en sus diseñadores. La afluencia de múltiples fenómenos y factores de todo tipo en un diseño espacial urbano hace complejo su estudio.

$\mathrm{La}$ arquitectura como ciencia no dedicaba conocimientos a esclarecer a profundidad, cuál es la influencia de esos espacios y cuáles son los comportamientos que se van haciendo recurrentes por los objetos que aparecen en ellos. La ciudad se imagina como espacio físico, quizás social, pero nunca como ahora, que se concibe como un actor de la educación. Muchas veces la relación se ve desde la formación del hombre para la convivencia en la ciudad, pero pocas veces se profundiza en como la ciudad educa a los ciudadanos. Por eso, tal como expone J. Fuentes (2019) es una necesidad incluir el punto de vista de la gente, contribuyendo al desarrollo de los conocimientos del espacio arquitectónico y sus aspectos simbólicos y epistémicos, entre el espacio construido y las personas que lo habitan.

En los espacios públicos aparecen reglas explícitas o no, que facilitan la valoración del comportamiento correcto o incorrecto. Esas reglas están asociadas a los objetos naturales o artificiales que aparecen en los espacios que regulan los comportamientos y al mismo tiempo contribuyen a la educación de las personas. Como expresa Salcedo (20110) la ciudad contiene una capacidad educadora que forma a sus habitantes.

De ahí que se esté creando una ciencia nueva denominada Pedagogía Urbana cuyo campo de conocimiento está incipiente. El avance de estos conocimientos podrá considerar el valor educativo de las ciudades que servirá como premisa para su diseño, también mostrará las diferencias de las prácticas sociales de los ciudadanos de diferentes ciudades.

En el estudio realizado se tiene como objetivo describir las características de dos lugares públicos que contienen objetos naturales y construidos y cómo son utilizados por las personas. Asimismo, refiere las insuficiencias de esos espacios para el uso que las personas les dan.

Por otra parte, se dan los resultados breves de observaciones sobre las características de una muestra de sujetos, sus comportamientos en el espacio público, el cruce de calles. En estos espacios, los ciudadanos no constituyen un grupo como tal, pero asumen roles y manifiestan otras particularidades que son interesantes. Generalmente, los ciudadanos no reciben conocimientos para comportarse en estos escenarios y, cuando los reciben, son muy elementales. Las reglas implícitas son trasladadas de generación en generación, por mecanismos de imitación, en esos espacios públicos. El comportamiento de las personas en espacios públicos tiene un sentido y un significado que expresa prácticas sociales habituales.

Esta parte del estudio, no tiene el propósito de transformar ni de realizar influencia educativa o pedagógica, sino mostrar el significado de los espacios y su relación con los comportamientos ciudadanos. Describir una parte de la realidad social que está presente en la ciudad y que resulta invisible en muchas ocasiones, pero que conforma la idiosincrasia de las personas de una nación.

\section{FORMACIÓN CIUDADANA Y COMPORTAMIENTO CIUDADANO}

La ciudadanía, el ser ciudadano, es un concepto que ha ido evolucionando a través de todas las épocas, hasta llegar a la actualidad en que se modifica la visión reduccionista de su contenido jurídico donde se evidencia la relación individuoestado, para dar más amplitud al término. Ahora se concibe esa ciudadanía como un proceso que está vinculado a toda la vida de la persona. Por eso se concibe en relación a todo el sistema de relaciones de la persona (Autor, 2016). Hasta hace poco, no se encontraban conceptos que la consideraran de esta forma, pero ya se van viendo estas consideraciones.

En el siglo XX se pone en cuestionamiento la vigencia de la ciudadanía desde el reconocimiento de derechos civiles y políticos y abre el espacio para un nuevo elemento en el análisis de la concepción de ciudadanía, los derechos sociales (Rincón, 2006). Se llega a acuñar el término de ciudadanía social (Cortina, 2008, citado por Gasca-Pliego y Olivera-García, 2011).

La propia evolución de los procesos políticos y sociales del capitalismo lleva más adelante 
a la afectación de los derechos sociales por lo que la ciudadanía se restringe a la elección de representantes y respeto mínimo a normas de convivencia (Rincón, 2006).

Pero no todos asumen la visión amplia del concepto, para algunos tiene limitantes, que deben ser profundizadas. Por tal razón, E. GascaPliego y J.C. Olivera-García (2011) expresan la necesidad de redefinirla para humanizarla y reflexionar sobre el respeto del otro. Ese otro, que empieza con los más cercanos, la comunidad, pero no termina sino en la humanidad (Autor, 2016). De ahí la necesidad de establecer bien las relaciones entre ciudadanía y nacionalidad.

Existen diferentes formas de entender el ciudadano y resulta un término no acabado y complejo en el que influyen diferentes ciencias. Asimismo, se puede hablar de cultura ciudadana en el sentido de cómo se regulan los comportamientos de los ciudadanos que hacen posible su convivencia. En el proceso de formación de la ciudad, según Elías (1989, citado por Rincón, 2006) se establecen signos y reglas para que los ciudadanos se apropien de elementos físicos y simbólicos de lo urbano, y así, adecuen su comportamiento para que puedan funcionar socialmente.

A. Mockus (2003) plantea que la regulación de los comportamientos de los ciudadanos es mediante tres sistemas de control social: la ley, la cultura y la moral. Esos tres sistemas deben tener congruencia para no dar lugar a contradicciones y conflictos en la educación.

M. Martín (2006) realiza un análisis de cómo la educación superior puede contribuir a la formación ciudadana y le da valor a la participación de los estudiantes que se expresa en la toma de decisiones de lo que les afecta y, por otra parte, la implicación del estudiante en la comunidad mediante actividades de aprendizaje académico con significado social, teniendo el territorio como fuente del mismo. Esto constituye una de las estrategias identificadas en otros estudios realizados con anterioridad (Autor, 2018).

La formación que la persona tiene en calidad de ciudadano se expresa en sus comportamientos en todos los escenarios públicos y en las diferentes situaciones de convivencia. Muchos de esos escenarios están en la ciudad. Las personas desde la niñez van asimilando formas de conducta que responden a las normas explícitas o no, de los espacios públicos que se encuentran en las diferentes ciudades. Al mismo tiempo, se ha venido desarrollando un enfoque de ciudad que profundiza en su valor educativo. Esta visión está dirigida a considerar cómo el contenido de las ciudades va condicionando los comportamientos, las significaciones, tradiciones y la identidad de las personas. La ciudad es vista como un microterritorio que tienen un sentido subjetivo vinculado a lo que en ella existe como la escuela, la casa, las áreas recreativas que frecuenta el individuo donde se configura la identidad individual y colectiva (Jaramillo, 2013, citado por García, 2019).

La profundización en el significado de la ciudad, así como la transformación que la misma ha tenido, ha llevado a la creación incipiente de una nueva ciencia que ha sido denominada Pedagogía Urbana dirigida al estudio de las prácticas educativas en la ciudad y como un campo de conocimientos que surge de las relaciones del individuo y el entorno urbano (Barrios y Fernández, 2008; Nájera, 2008; Páramo, 2009; Cuesta, 2010; Cotto, 2016). En sus inicios se veía como una disciplina formal incluida en la educación, pero en la actualidad se amplía hacia la concepción de la ciudad como agente educador.

Según Trilla (1993, citado por Cuesta 2010) se pueden identificar tres dimensiones de las relaciones educativas y el medio urbano: aprender en la ciudad, aprender de la ciudad y aprender la ciudad. Esta ciencia está relacionada con el medio ambiente, pero tiene su contenido específico, ya que el primero es esencialmente natural y el otro, generalmente construido. Pero esto no es absoluto porque los ríos, las lagunas, las plantas, los árboles, forman parte de ciudades y requieren comportamientos ciudadanos. Estas nuevas concepciones llevarán a reflexionar acerca de las relaciones educación- sociedad.

Estas consideraciones se hacen a partir de la idea de que la ciudad civiliza a las personas, que socializa normas, experiencias, saberes a través de todos sus elementos componentes y que es un objeto de conocimiento que va más allá de la superficie que la delimita, del aprendizaje directo que se manifiesta.

F. Lezcano (2012) realiza un análisis necesario para la interpretación de las ideas actuales referidas a la ciudad. Al hablar de ciudadano existen dos campos semánticos: uno estrecho, que expresa un status y se relaciona con la pertenencia a un colectivo o país y que de esta manera es un concepto excluyente de otros países, del área rural o de otros pueblos. Esta visión se apega más 
a los inicios del término que sólo consideraba a las minorías. Otro es más amplio, que identifica al habitante de una ciudad, estado, país. En este caso está muy ligado a nacionalidad.

J. Acosta (2019) resalta, con acierto, que la ciudad es un espacio físico con vida propia, que pasa por un proceso desde que surge hasta que se desarrolla donde influyen factores económicos, sociales, culturales, que están presentes $\mathrm{y}$, dificultan y complejizan su análisis. La ciudad es construida por los hombres y a su vez ella va construyendo al ser humano. La ciudad está llena de contenidos que forman a los ciudadanos culturalmente a partir de las relaciones que se establecen con esos contenidos (Cotto, 2016). Por eso, la ciudad se convierte en un espacio de formación, formal e informal, donde las reglas juegan un papel importante.

Como se ha expresado, la ciudadanía es considerada un proceso que, dada la globalización y la evolución histórica de los procesos políticos y sociales, requiere la profundización para que incorpore al hombre como ciudadano del mundo. Ahora la persona no puede limitarse a sus círculos más reducidos de relaciones, ni siquiera a su país. Sus vínculos se han ampliado de manera significativa y esto conlleva la asimilación de comportamientos que van desde lo más cercano hasta lo mundial.

Los ciudadanos están relacionados con el tipo de hombre que habita la sociedad y que interactúa con los espacios, los objetos naturales y físicos de los mismos y las personas y que, con sus prácticas ciudadanas satisfacen sus necesidades sin afectar la convivencia tanto de la colectividad como de la humanidad. Tratar de acceder a esas prácticas y sus significados mediante observaciones en los espacios públicos contribuye a enriquecer todo lo que en un futuro pudiera ser la Pedagogía Urbana como ciencia reconocida.

\section{COMPORTAMIENTO CIUDADANO EN LOS ESPACIOS PÚBLICOS}

Los estudios arquitectónicos han dado un cambio que constituye un reto para esa ciencia. La tendencia va cada vez más a alejarse del valor de la combinación espacio y objeto arquitectónico. En la actualidad tiene una importancia vital la organización de las formas arquitectónicas en el espacio, en el que habitan, conviven y existen las personas. Esto ha llevado a tener en cuenta la relación de lo que se construye con lo que las personas necesitan hacer y hacen en esas construcciones como parte de su cotidianidad. Para J. Fuentes (2019) se manifiesta una creciente complejidad en los procesos constructivos porque hay que incorporar la subjetividad humana: los significados que tienen para las personas, la cultura simbólica, las interacciones que se dan en ellos. Es una interrelación complicada entre lo cualitativo y lo constructivo.

Esta tendencia ha influido en que se comiencen a realizar estudios sobre la influencia de los espacios arquitectónicos en las personas y cómo las reacciones que generan influyen en las conductas. Es una interinfluencia entre el ambiente y las personas que ha dado lugar a una ciencia, también incipiente, denominada Neuroarquitectura (Tlapalamatl, 2019).

Por tanto, el espacio incluye aspectos simbólicos y sociales que no pueden dejar de tenerse en cuenta. Es en los espacios públicos donde se expresa claramente la relación de sus formas con la vida que se realiza en esos espacios. Dentro de este término, como refiere J. Fuentes (2019), se consideran las plazas, los jardines, los parques, las calles, etc. donde se manifiesta la traza urbana. En esa traza urbana están los comportamientos ciudadanos que expresan las formas de actuar de las personas, entre sí y con el ambiente externo. Comportamientos que se evocan tanto desde el interior de las personas como desde el propio ambiente con su escenario y organización. Esos comportamientos dan una visión de cómo están formadas, en relación a su rol de ciudadanos. De ahí que resulte interesante estudiar las reglas de convivencia en espacios públicos donde las personas tienen relaciones transitorias y cómo se implican en las que están presente. En el espacio público transitorio hay diversidad de roles que se entremezclan y aunque no es posible detallarlos, las personas los van adecuando a la situación.

Como bien analiza O.J. Cuesta (2010) en esos espacios se da el aprendizaje situado. Dadas las transformaciones que se producen en el mundo, es un espacio que hay que tener en cuenta, ya que va adquiriendo mucha importancia por los procesos informales que se facilitan en él.

Según Saladrigas (1997, citado por Cuesta, 2010) el espacio público es el escenario que es de dominio de todos, como un gran terreno donde la ciudadanía se reconoce a sí misma. El espacio público, para Páramo (2007, citado por Cuesta, 
2010) son áreas accesibles para todos, donde las personas no tienen muchas restricciones. El propio Cuesta (2010) considera que independientemente de cualquier conceptualización, no debe omitirse la formación e interacción social que allí se da, la expresión de múltiples cosmovisiones y el aprendizaje de reglas.

\section{LOS CRUCES DE CALLES COMO ESPACIO PÚBLICO}

Tradicionalmente se concibe la formación de las personas en lugares tradicionales como la casa, la familia, la escuela, pero son pocos los estudios que revelan el papel de los espacios públicos en la subjetividad humana. No obstante, por la vivencia y la experiencia es conocido cuanto de aprendizaje tienen ellos, considerados con la denominación de la calle. A modo de graficar, se conoce de los niños que en muchos países de América viven en la calle y se buscan la vida en ella. No tienen educación, pero han adquirido una riqueza de métodos naturales, formas de expresarse, técnicas de influencia, razonamientos sagaces que fueron adquiridos rodando en las calles, cuestiones que no aparecen en ningún currículo ni programa de estudio.

El espacio público se constituye en una fuente de desarrollo subjetivo y en un escenario en que se muestran acciones y comportamientos que son accesibles a la observación y al registro, para su estudio. Uno de los espacios públicos que presentan una riqueza innegable son los cruces de calles. Son espacios de un uso significativo, aunque constituyen tránsito de las personas. La estadía de las personas en ellos es limitada y generalmente las acciones no permiten profundizar en detalle, pero dan un conjunto de elementos que sirven para conocer no solo la influencia de las personas en ellos sino también, lo contrario.

En ese breve espacio del cruce de calles, se da una interrelación entre las personas y todo el conjunto de elementos que están presentes en ese espacio y la diversidad de usos vinculados a la satisfacción de las necesidades del ciudadano y de la vida cotidiana.

Los cruces de calles son espacios en los cuales las personas se desenvuelven con fragmentos de su modo de vida, con las interpretaciones que hacen de las acciones de los demás y con el significado que les dan a los objetos que aparecen en esos cruces. En los cruces de calles las personas conocen los usos de los diferentes objetos y cuáles son las conductas esperadas por sí mismo y por los demás. Existen en la ciudadanía modos habituales de interpretar. Por otra parte, también juegan un papel importante los elementos del lenguaje no verbal que permiten interpretar las conductas de los demás. Ese es un aprendizaje producto de las experiencias y la socialización de la vida en esos espacios públicos. Todo esto es lo que permite las relaciones con la colectividad y la convivencia con los demás.

En esos espacios públicos se diluyen las diferencias entre las personas. En el cruce de calles, en el conjunto de personas, ellas son relativamente iguales porque se diluyen las particularidades específicas y todos se insertan en el espacio con roles del propio espacio. Todas, de manera transitoria, se involucran en un entramado en el que asumen determinados comportamientos, en los cuales inciden un conjunto de señales de diferentes tipos que los regulan. Todos los objetos de ese espacio, construidos o no, condicionan las conductas de las personas. Solo penetrando esas interrelaciones pudieran encontrarse las particularidades de esas personas.

Como todo espacio público, en los cruces de calles, existen las reglas, que pueden ser de diferentes tipos. Según A. Milena Burbano "las reglas son descripciones verbales que ofrecen la oportunidad de relacionarse de forma apropiada con el entorno, otras personas u objetos a partir de las consecuencias que anuncian sobre el ambiente" y pueden ser morales, sociales y jurídicas (Milena, 2009,30). Ellas surgen por el uso natural de las personas o establecidas formalmente en leyes, manuales, etc. Las reglas facilitan la valoración de la acción correcta e incorrecta. Muchas están asociadas a señales y objetos construidos que están incorporados al espacio público. Ellas implican comportamientos específicos de las personas, por lo que además de regularlos, favorecen la educación, generando formas de comportarse los ciudadanos.

M. A. Salcedo (2010) realiza un estudio exhaustivo de las señales que aparecen en las calles y su papel regulador en la conducta de las personas. Las señales trascienden la interpretación jurídica de ellas. Pueden ser: señales urbanas que regulan el desplazamiento, marcadores de trayectoria, direcciones, reguladores de conducta, de advertencia, comerciales, publicitarias, indicadoras de lugar, gráficas e institucionales. 
No solamente las personas que se encuentran en los cruces de calles son los transeúntes, porque aparecen otras. Allí afloran trabajadores formales y no formales, tanto estatales como no estatales. Componen el lugar diferentes objetos constructivos que pueden ser viviendas, comercios, servicios, edificios, y otros, con significados y conductas esperadas. No siempre la estética de ellos satisface el gusto de los usuarios y también se dan comportamientos que deslucen el espacio. Todo ese conjunto con un significado y una influencia sobre las personas, va enriqueciendo las prácticas de ciudadanía.

\section{COMPORTAMIENTO CIUDADANO EN CRUCES DE CALLES}

El estudio fue realizado en dos períodos, de un mes cada uno, a finales del 2019 y principios del 2020. Se lleva a cabo por 12 observadores que fueron capacitados previamente, tanto para la temática como para el uso del método de la observación. Este método se utiliza en el tipo de observación natural, no participativa o externa, directa, con registro de diario de campo y guías para procesamiento de datos.

El total de observaciones fueron 100, distribuyéndose 50 en cada uno de los espacios estudiados, lo que da lugar a un total de 50 sujetos estudiados en cada cruce de calles y un número considerable de observaciones de indicadores. Los sujetos se seleccionan al azar. Se observaron dos espacios públicos: el cruce de calle L y la calle 23 en el centro del Vedado, Municipio Plaza de la Revolución, La Habana y el otro espacio, lo conforma el cruce de calles Ave de los Presidentes (G) y la calle 27, en el Vedado, Municipio Plaza de la Revolución, La Habana. Ambos escenarios se caracterizan por ser concurridos durante casi todo el día.

Un valor adicional que tiene el estudio es que con estas observaciones hay un acercamiento a la idiosincrasia de los ciudadanos de La Habana. Como es sabido, en esta ciudad la mayoría de las personas se trasladan en transporte público a diferencia de otras ciudades del mundo en que no pueden tomarse en cuenta dichas observaciones para ilustrar las características típicas de las personas.

El cruce de las calles L y 23, toma como punto de referencia el paradero de los ómnibus que se encuentra delante de la heladería Coppelia, teniendo al frente un Hotel en construcción en el momento de la observación, a la izquierda el cine Yara y a la derecha, separado las oficinas de la AIN.

En ese espacio se encuentran diferentes objetos sociales. Entre ellos: objetos construidos: como 9 viviendas, 7 centros comerciales y de servicios, 1 centro de trabajo, 1 paradero de ómnibus, 4 pasos peatonales, 1 escalera de acceso a servicio. Objetos naturales: 4 jardines en centros de comercio y servicio. En este escenario se encuentran numerosos comerciantes informales de alimentos y otros artículos, los que ascienden a más de 20. Estos permanecen en el lugar durante un período de tiempo en el día y se retiran, por lo que de noche es un área más desierta. Existe un obstáculo para la movilidad que lo constituye una valla larga que abarca todo el espacio en la acera de enfrente donde se construye el hotel. Esto hace que muchas personas caminen a veces por la orilla de la calle, lo cual puede ser peligroso.

Aunque el lugar tiene relativamente facilidad espacial, el volumen de transeúntes provoca que, entre los objetos construidos y la movilidad, dé un alto grado de posibilidad de contacto físico entre las personas. A esto también contribuyen los comerciantes informales. Sus aceras son amplias y en algunos tramos tiene el espacio verde hasta la calle. El paradero no es tan grande, aunque tiene espacio detrás, que le da capacidad de maniobra a las personas para cualquier eventualidad. Además, tiene una vía no vial que termina en el paradero. La práctica ciudadana ha modificado el uso social del mismo, ya que no es el lugar donde se realiza la espera de los ómnibus. La intersección de este cruce tiene una variedad de rutas de ómnibus que irradian el resto de los Municipios, es una arteria central de transportación y tiene prohibido el paso de vehículos de alto tonelaje. Esta situación y la existencia de la demandada heladería Coppelia, hace que se entremezclen los transeúntes y los usuarios de la heladería, formando gran aglomeración y reducción de la distancia social entre las personas. En todo el frente de la heladería existe una reja que delimita la propiedad estatal, ella está construida en el límite de la acera, lo que constituye un área de exclusión. Por esta razón, no existe zona de seguridad entre la acera y la reja, cosa que no permite la movilidad de la cantidad de personas, en caso de emergencia. La reja podría alejarse de la acera para dejar un espacio de seguridad, ya que los jardines tienen área suficiente para ello. Los árboles que están sembrados en canteros de 
las aceras, son de mediano tamaño por lo que no afectan la visión de todo el escenario público.

Existen señales visuales urbanas con función normalizadora de las acciones de los ciudadanos y el movimiento de las personas, como lo son las cebras en los pasos peatonales, los semáforos peatonales, tablillas de rutas de omnibus. La relación entre los objetos y las prácticas ciudadanas se corresponden, ya que se hace uso de la cebra, del semáforo, excepto del paradero. En este espacio existen cestos de basura en tramos específicos de las aceras. No obstante, la existencia de tanto comercio informal ha contribuido a que las personas se habitúen a detenerse para hacer compras, utilizar los servicios y se configure un tránsito constante entre los ciudadanos. Asimismo, se presentan prácticas como conversar, crear grupos, pasear en familia, aun cuando el lugar no esté previsto para ello. Quizás estas prácticas han dado lugar a un proyecto, comenzado, en el área de estacionamiento detrás del paradero, que es amplia, y en la cual aparecen algunas bancas para descanso y en ocasiones, kioscos de ventas. Otra práctica ciudadana que se manifiesta, que se considera rechazable, pero se percibe en alguna medida, es el cruce de la calle fuera de la cebra y sin el respeto del semáforo, lo cual aún no es habitual.

Es un área tan céntrica, que no puede dejar de considerar la existencia de objetos de identificación de la ciudad y el país. En la propia acera están incrustadas obras reproducidas de cuadros de pintores cubanos reconocidos, en postes adecuados existen colgadas fotografías grandes de lugares de La Habana en conmemoración a los 500 años, no se visualiza la bandera como símbolo de identidad esencial.

Uno de los edificios más destacado en este lugar, es el Hotel Habana Libre (antes Havana Hilton). Es uno de los objetos construido, que goza de prestigio y distinguido por su ubicación en este céntrico lugar. Posteriormente, ganaron más notoriedad los que se ubicaron en la Habana Vieja, casco histórico de la ciudad, pero el Habana Libre aún conserva su valor. En sus salones se celebraron acontecimientos importantes a los que asistieron personas destacadas en lo social, lo intelectual y lo político. Uno de los objetos valiosos lo constituye el gigantesco mural que aparece en su fachada principal del hotel, creado por la pintora cubana Amelia Peláez. Ese mural ha tenido que ser restaurado y constituye un objeto de imagen de la ciudad y valor patrimonial. No obstante, en esa intercepción del cruce, la acera se estrecha un poco lo que obstaculiza la movilidad.

En cuanto al cumplimiento de reglas apreciadas en las observaciones realizadas a los 50 sujetos, se pueden considerar: leyes del tránsito (cruzar con semáforo, por la cebra), utilizar cestos basura, uso adecuado de los objetos del espacio público, no comer, ni fumar, ni ingerir bebidas alcohólicas en la calle, hablar en tono adecuado, vestir apropiadamente, se encontró un $87 \%$. El resto de las observaciones se ubican en el incumplimiento de reglas, que pueden ser lo contrario de las anteriores y otras. Entre las que se incumplen están: no caminar por la acera, cruzar sin respeto a las leyes del tránsito ( $\sin$ respeto a la cebra o al semáforo), no utilizar los cestos de basura, no pedir permiso al pasar, caminar utilizando celular, ingerir bebida, comer en la vía, no respetar el orden en la fila.

Lamentablemente, aunque el por ciento que incumple las reglas de comportamiento ciudadano de los sujetos observados, tanto explícitas como implícitas, no es elevado, basta para que se dé una imagen desfavorable de la ciudad. A esto se une que este tipo de conducta, que es sancionada socialmente, cobra más visibilidad dentro de las prácticas ciudadanas.

Las observaciones realizadas de forma individual a los 50 sujetos, dan otra información también interesante. A pesar de haber en el país una mezcla de color de la piel de sus ciudadanos, donde se dan tanto el color blanco, como el negro y el mestizo, el $58 \%$ de ciudadanos que transitan este cruce son de color blanco, el 20\% es de color mestizo y $22 \%$ es de piel negra. Es decir, que hay un ligero predominio de la piel blanca.

En cuanto al sexo de los sujetos, se observa un $60 \%$ de sexo femenino, un $46 \%$ de jóvenes que predominan en las horas de la tarde; mientras que el adulto tiene un $36 \%$ y predomina en horas de la mañana.

Con relación a las observaciones realizadas al vestuario, se encuentra que sólo un $5 \%$ puede considerarse que lo presenta sucio y un $4 \%$ roto. Se puede valorar que constituyen comportamientos no apropiados para estos espacios. El 23\% de las observaciones muestra como prenda superior la camiseta o el desmangado, que es lógico en un país de eterno verano. Un $38 \%$ de las observaciones muestran pantalones o jean o shorts como prenda inferior. Sólo el 13\% viste vestido muy corto. El $52 \%$ de las observaciones muestran que se 
usan adornos, joyas, aditamentos. Se valora como una práctica ciudadana de la imagen de esta ciudad. Entre los objetos adicionales que tienen las personas, las observaciones dan que el 19\% lleva carteras e igual por ciento bolsas de nylon, un 13\% mochilas, sólo un 2\% lleva laptop. En este sentido, no existe nada que caracterice al ciudadano que transita porque puede llevar diferentes objetos. Si es algo a distinguir que no se observa llevar consigo la laptop, esto puede deberse a no ser habitual su uso en las tareas cotidianas o en los lugares donde permanecen las personas. Independientemente que las tecnologías no son un objeto accesible para todo el mundo.

En cuanto al andar no hay diferencias en si es lento o rápido porque se distribuyen equitativamente. las observaciones. Un $40 \%$ avanza con otras personas y un $42 \%$ de las observaciones muestran que conversan; en cuanto al tono de voz se observó un $35 \%$ habla en voz alta o muy alta. Con relación a la utilización del espacio entre las personas, un $28 \%$ toca al interlocutor, un 36\% se aproxima y un $36 \%$ mantiene la distancia social y personal.

En estas últimas observaciones se aprecian características idiosincráticas del nacional que es sociable y abierto, generalmente habla en voz alta y lo hace con cercanía a las personas, dándole una utilización amplia al espacio físico y también al espacio sonoro. Es una persona muy expresiva tanto en sus movimientos gestuales (mímica y pantomima) como en los elementos paralingüísticos (mímica "vocal" y "visual") y extralingüísticos.

El cruce de calles de Ave de los Presidentes $y$ calle 27 es un espacio público concurrido, pero no de igual magnitud que el anterior. En él, no permanecen tanto estacionadas, pero sí en movimiento, se observan muchas personas. Es un lugar caracterizado por la presencia de varios hospitales especializados que le rodean. El punto de referencia es el paradero que existe en la Ave de los Presidentes en dirección hacia la Plaza de la Revolución. También tiene muy cercana la Universidad de La Habana, especialmente sus facultades de Arte y Literatura, Química, Física, el Centro de Estudios de Administración y Dirección y, el Estadio Universitario. Este es un cruce de calles que conecta muchas ciudades de La Habana, por lo que por ella transitan muchos ómnibus urbanos.

Como objetos construidos en el espacio están 4 casas, 2 comercios y servicios formales (entre ellos un expendio de gasolina), 3 cafeterías,
2 centros de trabajo, 1 parque, 1 residencia estudiantil y un monumento. Es un cruce de calles con tres áreas de parada de ómnibus, pero solo una tiene construido el paradero. Éste tiene detrás un área verde que garantiza la seguridad de la movilidad en casos de emergencia. Esa área topa con un gran parqueo de vehículos y algunas áreas de descanso de las personas, con asientos para ello. La avenida frente al paradero es de doble vía y con varios carriles de tránsito de vehículos. Es la denominada Avenida de los Presidentes ya que en todo su recorrido se encuentran monumentos a presidentes de diferentes países, que han realizado una actividad meritoria por sus pueblos. Frente al paradero está construido un monumento de un tamaño considerable dedicado al presidente José Miguel Gómez que gobernó el país en 1909, la obra es de corte neoclásico y ha permanecido a pesar del tiempo. En la otra senda de la avenida está ubicada una parte del Hospital Docente Calixto García, primero en vincularse a la docencia universitaria y con larga tradición de servicio verdaderamente humanitario.

Entre los objetos naturales se encuentran jardines, áreas verdes y árboles que bordean las aceras externas, son enormes y bellos los sauces llorones del que descienden largos bejucos que semejan lágrimas que caen; de ahí su nombre. También el cruce tiene un espacio construido que separa las dos vías, que constituye una franja de seguridad y ornamental de la avenida. En esta franja se ubican asientos públicos para el descanso y recreación de las personas. Aunque esos objetos indican la pertinencia de la práctica ciudadana de descansar y recrearse, ellos no son tan utilizados por las personas. Solo se ven, esporádicamente, algunas personas disfrutando de ese lugar. No se observan suficientes cestos de basura en correspondencia con las personas que transitan.

Como se ha expresado, sólo uno de los lugares para tomar el ómnibus tiene paradero, los otros dos se tiene como práctica esperar en la acera, que no son amplias, por lo que se aglomeran personas en diferentes filas para diversos recorridos. Tampoco existe protección en ellas para el sol o la lluvia, el terreno es muy irregular por las raíces de los grandes árboles que allí se encuentran, lo que constituye un peligro para las personas, especialmente las adultas mayores. En este espacio existen señales para la regulación del comportamiento de las personas como las señalizaciones del tránsito. El paso peatonal que 
existe está muy alejado de las áreas de paradas por lo que su función es limitada, no dando protección a la mayor cantidad de transeúntes de este espacio, no existe tampoco semáforos en esta área, solo los gráficos del sentido de las vías y señales de velocidad permitida. Esto hace que se haya reafirmado la práctica de cruzar mirando a los dos lados, se apresuren las personas y algunas corran. En el monumento tampoco existen señales para las prácticas ciudadanas, por lo que las personas se sientan en las escaleras, los niños patinan, lo que es contrario a las conductas aceptables. Las personas también utilizan los comercios y servicios que allí se encuentran.

Por las observaciones realizadas se tiene que un $89 \%$ cumple las reglas, tanto explícitas como implícitas. Las reglas que según las observaciones se cumplen son: hablar en voz baja, no pisar el césped, no arrojar basura en la calle, respetar la fila de espera, no dañar los bienes públicos, atender al cruzar la calle, pedir permiso al adelantar a otra persona. Entre las reglas que aparecen incumplidas están, en alguna medida, hablar en voz alta, arrojar basura a la calle, ingerir bebidas alcohólicas, distraerse al cruzar la calle, uso inapropiado de los objetos con letreros, hacer pregones en voz muy alta.

Este cruce de calles es muy agradable y tiene la belleza que le da el verdor de sus áreas. $\mathrm{Su}$ amplitud permite tener visibilidad hacia un paisaje atractivo que concluye en el malecón habanero, donde se percibe el mar.

En las 50 observaciones realizadas se encuentra un $60 \%$ de sujetos con color de la piel blanca, un $20 \%$ de color mestizo y un $20 \%$ de piel negra. Se aprecia que de igual forma hay un valor superior en las personas blancas, no obstante, la idiosincrasia del nacional, que es mezclada. Un $72 \%$ son del sexo femenino, un $28 \%$ son jóvenes, $38 \%$ son adultos y $14 \%$ son adultos mayores.

Sobre las observaciones a la vestimenta, se encuentra que el $18 \%$ utiliza la camiseta como prenda superior, $12 \%$ viste short como prenda inferior. Un $6 \%$ presenta sucia la ropa y un $10 \%$ rota. Un sujeto se encontraba descalzo. Estas constituyen prácticas ciudadanas rechazables por no cumplir normas implícitas de urbanidad. Se observa que el $12 \%$ muestra vestido largo, más allá de la media pierna y solo el $6 \%$ viste muy corto. Un 36\% tiene como prenda inferior el pantalón o jean. Aquí se refleja que la mayoría de las personas cuidan su imagen y aspecto personal, así como la higiene.
Con relación a los objetos personales ornamentales se observa que un $62 \%$ tiene aditamentos, joyas, espejuelos. Entre los objetos que les acompañan el mayor por ciento es de las mochilas, con un $36 \%$. Le sigue un $26 \%$ con carteras y un $14 \%$ con bolsas de nylon. Las laptops aparecen solo con un $8 \%$. En la mayoría de las observaciones aparece un objeto acompañante. En este cruce aumenta el uso de la laptop, lo que puede estar justificado por la cercanía de las instituciones de la Universidad de La Habana.

El $50 \%$ de las personas están conversando con otros. En cuanto al celular, el $30 \%$ usa el celular. Es significativo que un $12 \%$ de sujetos ingiere bebidas alcohólicas y 16\% fuma. Estas son conductas rechazables. En cuanto a la bebida quizás sean promovidas por la percepción del lugar amplio o con condiciones de recreación, no obstante, es inadecuado. En cuanto al hábito de fumar, en realidad el por ciento es bajo, tomando en consideración que en este país ese hábito era elevadísimo en cualquier edad. Se debe tener en cuenta que es el mejor cultivador de tabaco del mundo, codiciado por todos los países, sin que medien buenas o malas relaciones diplomáticas. Pero las campañas han sido muy fuertes y ha disminuido mucho el hábito nocivo de fumar, pero todavía se puede observar.

En la velocidad al caminar, los por cientos están equiparados en lento y rápido por lo que no se toma como característica. Un $28 \%$ habla en voz muy alta o alta y en la utilización del espacio se observa que un $18 \%$ toca al interlocutor y un $36 \%$ se aproxima, solo un $2 \%$ mantiene la distancia social adecuada. Esto como se ha visto es parte de la idiosincrasia de los nacionales. Se conoce que es efusivo, expresivo y abierto en todas las expresiones del lenguaje tanto verbal como no verbal.

\section{CONCLUSIONES}

El concepto de ciudadano es necesario verlo en la actualidad con una visión más amplia, lo que permite orientar mejor la formación. El hombre es un ser social, que vive en sociedad y necesita de los demás par su existencia. Esto obliga a realizar prácticas ciudadanas de convivencia en todos los espacios públicos.

La ciudad está llena de espacios, de objetos naturales y construidos que ejercen una influencia educativa en las personas y modifican sus subjetividades. Se habla por algunos autores de 
tres dimensiones de la relación educativa y el medio urbano: aprender en la ciudad, aprender de la ciudad y aprender la ciudad.

De ahí que aparezcan nuevas ciencias como la Pedagogía Urbana y la Neuroarquitectura y que, por otra parte, hayan cambiado los conceptos arquitectónicos para tener en cuenta más al ser humano y el uso que hará de esos espacios.

Los cruces de calles constituyen espacios públicos, no completamente estudiados, a donde concurren muchas personas, en los que se da la equiparación, se diluyen las desigualdades, se expresa la cooperación, se adoptan roles transitorios que llevan a comportamientos específicos que se van asimilando por los ciudadanos. Es un escenario de interacción entre las formas urbanas y las prácticas sociales.

Los cruces de calles de La Habana que fueron estudiados, muestran tanto sus fortalezas como las debilidades que aún mantienen y que requieren modificaciones de tipo arquitectónica y urbanística. En las observaciones realizadas se puede considerar cómo se expresa el espacio, con todos sus objetos, en determinadas prácticas ciudadanas. La sociedad cubana es muy compleja tanto por su origen como por la población que la compone. Tiene características que le son favorables como no tener diversidad de etnias ni culturas, ser pequeña su población, haber disminuido las diferencias sociales, no tener analfabetismo, contar con la garantía de algunos esenciales derechos sociales, todo ello contribuye a dar homogeneidad a su ciudadanía y un contenido específico a la subjetividad.

Por las observaciones realizadas se puede valorar que en su mayoría los ciudadanos estudiados tienen un comportamiento acorde a reglas urbanas, gran parte de los sujetos mantienen posturas favorables a la comunidad. Esto no niega que se observan sujetos que tienen comportamientos rechazables desde el punto de vista ciudadano y que en sus prácticas muestran que necesitan aumentar su formación en este sentido.

$\mathrm{Si}$ se logra una mayor conciencia, una influencia mayor de la ciudad para todos sus habitantes, La Habana podría ser mucho más real y maravillosa de lo que es en la actualidad. C

\section{REFERENCIAS BIBLIOGRÁFICAS}

Gónzalez, R. (2016). “Cap. 12 Acerca del concepto de formación ciudadana". En V. Ojalvo e Y. Cortiza (Coords.) La Responsabilidad Social Universitaria, paradigma de la nueva universidad. México: Editorial Juárez del Estado de Durango. 1ra edición, pp. 269-282 ISBN: 978-607-503-185-9

Gónzalez, R. (2018). "Las estrategias educativas para la formación ciudadana según su alcance y sentido". En Revista Perspectivas. Núm. 17, pp. 1-18 DOI https//doi. org/10.15359/rp.17.3

Barrio, J. L. y Fernández, J. D. (2007). "La Pedagogía Urbana como experiencia educativa en adultos". En Pulso. Núm. 30, pp. 171-190

Cotto, L. (2016). "La pedagogía urbana, un camino posible y necesario para las ciudades". 80 grados, en Prensa sin Prisa. http:// www.80grados.net/la-pedagogia-urbanaun-camino-posible-y-necesario-para-lasciudades/ (Consultado 20 de marzo 2020)

Cuesta, O. J. (2010). "Pedagogía urbana, convivencia ciudadana y aprendizaje por reglas". En Revista Educación y Desarrollo Social. Núm. 2, pp. 176-188

Fuentes, J. (2019). "Espacio público y vida cotidiana: hacia un cambio de teorías en diseño urbano y arquitectónico". En Revista Contexto, Vol. XIII, núm. 19, pp. 39-47

García, A. (2019). "Ensenada imaginada: representaciones urbanas desde la niñez”. En Revista Contexto, Vol. XIII, núm. 19, pp. 89-100

Gasca-Pliego, E. y Olvera-García, J. (2011). "Construir ciudadanía desde las universidades, responsabilidad social universitaria y desafíos ante el siglo XXI". En Revista Convergencia, Revista de Ciencias Sociales. Vol. 18, núm. 56, mayo-agosto, 2011, pp. 37-58

Lezcano, F. (2012). "Concepto de ciudadano, ciudadanía y civismo". En Revista de la Universidad Bolivariana, Vol. 11, núm. 32, pp. 269-304

Martín, M. (2006). "Formación para la ciudadanía y educación superior". En Revista Interamericana de Educación. Núm. 42

Milena, A. (2009). "La convivencia ciudadana: su 
análisis a partir del "aprendizaje de reglas". En Revista Colombiana de Educación. Núm. 57, pp.28-45

Mockus, A. (2003). "Cultura ciudadana y comunicación". En Revista La Tadeo, Núm. 8, pp. 106-111. www.utadeo.edu.co. Consultado 24 de abril 2020

Nájera, E. (2008). "Esbozos para una pedagogía urbana pertinente a los desarrollos educativos en las ciudades". En Polis [En línea], 20 | 2008, Publicado el 19 julio 2012, consultado el 15 abril 2020. URL: http://journals.openedition.org/polis/3421

Páramo, P. (2009). "Pedagogía urbana: elementos para su delimitación como campo del conocimiento". Ensayos. En Revista colombiana de educación, Núm. 57

Rincón, M.T. (2006). "Cultura ciudadana, ciudadanía y trabajo social”. En Revista Prospectiva, Núm. 11, pp. 45-65

Salcedo, M. A. (Comp.) (2010). Educación, identidad y comportamiento ciudadano en el espacio público. Cali: Editorial Bonaventuriana. Universidad de San Buenaventura, Colombia.

Tlapalamatl, E. (2019). "La arquitectura como producto del cerebro". En Revista Contexto, Vol. XIII, núm. 19, pp. 61-72 